\title{
LINE X TESTER ANALYSIS FOR YIELD AND ITS TRAITS IN BREAD WHEAT
}

\author{
ABDEL NOUR, NADYA A. R. ${ }^{1}$, HAYAM S.A.EL-FATEH ${ }^{2}$ AND A.K.MOSTAFA ${ }^{1}$ \\ 1. Wheat Research Dep., Field Crops Research Institute, ARC, Giza, Egypt. \\ 2. Lab. for Design and Statistical Analysis Research, ARC. Giza.
}

(Manuscript received 3 March 2011)

\begin{abstract}
The present study was carried out in three successive seasons from 2007 to 2010.The first and second seasons were at El-Giza Research Station while the third season was at Kafr El- Hamam Research Station. The main objective of this study was to estimate combining ability variances and effects of eighteen wheat genotypes which were crossed to three local wheat cultivars, i.e., Gemmiza 9, Sakha 93 and Giza 169 as testers using Line $\mathrm{x}$ Tester analysis in $\mathrm{F} 1$ and $\mathrm{F} 2$ populations for grain yield and its components. The differences among genotypes, parents and crosses were highly significant for all studied characters. Both general and specific combining ability variances were highly significant for all characters. The results revealed that non additive gene effects were larger than those of the additive ones and played the major role in the inheritance of all studied characters. Also, the maximum contribution to total variance of grain yield per plant and other traits was made by female lines. The parental lines 3, 4, 14 and 15 proved to have better general combiners for grain yield per plant and most of its components in grain yield improvement. Hence, four female parents could be used in future programs to improve grain yield. Tester number 1 appeared to have better general combiner for grain yield per plant and some other traits. These parents presumed to have a relatively large number of favorable alleles for these traits. However, the hybrids (L3 X T3), (L4 X T1), (L14 X T2) and (L15 X T2) were excellent harmonious combinars for specific combining ability effects for all characters including grain yield per plant. Hence, it would result in transgressive segregants for grain yield per plant in late segregating generations due to the predominant non - additive gene action.
\end{abstract}

Key Words: Genotypes, Combining ability, Gene action

\section{INTRODUCTION}

Bread wheat (Triticum aestivum L.) is a major food crop in the world. In Egypt, it is used as a stable food grain for urban, rural and bediwin societies and as a major source of straw for animal feedins. However, geometrical increase in the Egyptian population has been a challenge for agricultural scientists. To feed burgeoning population of Egypt, there is a dire need to develop wheat genotypes having high yield potential. This could be achieved by exploring maximum genetic potential from available wheat germplasm. Breeders should concentrate on development of productive wheat varieties by crossing good general combining lines for grain yield and selecting transgressive segregnts from resulting hybrids. Combining 
ability analysis gives very useful information with regard to selection of parents based on the performance of their hybrids. Moreover, this analysis helps the breeders to identify the best combiners which may be hybridized either to exploit heterosis or to build up the favorable fixable genes. In the initial stages of breeding program the breeders need general knowledge about gene action and genetic system controlling the genetic variation of the studied characters. The line $X$ tester analysis was used to estimate both general combining ability effects (G.C.A.) and specific combining ability ones (S.C.A.) for yield and its components and other important agronomic traits of wheat by several authors. (Hassan and Abd El- Moniem (1991); Salem and Hassan (1991) Singh et al. (1994); Gupta and Ahmed (1995) and Rajara and Maheshwari (1996); Masood and Kronstad (2000); Singh et al. (2000); Ahmed et al. (2003) and Joshi et. al. (2003)) reported that majority of genetic variances of grain yield as well as yield components were under control of additive nature of genes. On the other hand, Suleyman and Akguni (2007), observed that grain yield per plant and most of the traits were under control of non- additive gene effects. In another study, the magnitude of additive genetic variance was higher than non - additive variance for all studied characters.

The present study was designed to find out good general combining lines, testers and crosses for various characters so that suitable crosses are marked in view of gene action for adopting proper selection method to develop productive wheat cultivars.

\section{MATERIALS AND METHODS}

The present study was carried out at El - Giza Research Station, Egypt during 2007/ 2008and 2008 / 2009 seasons. In 2009 / 2010 season, the final experiment was conducted at Kafer El - Hamam, El -Sharkya, Egypt. In 2007/ 2008 season, line x tester mating design was performed through 18 genotypes (lines) in addition to three testers to produce the hybrid seeds of 54 crosses. Parents of the be forementioned genotypes are listed in Table 1. 
Table 1. The name and pedigrees of the parental genotypes.

\begin{tabular}{|c|c|c|c|}
\hline Genotype & \multirow{2}{*}{ Name } & \multirow{2}{*}{ Pedigree } & \multirow{2}{*}{ Origin } \\
\hline Lines $(L)$ & & & \\
\hline 1 & Anza & $\mathrm{LR} / \mathrm{NIOB} / / 3^{*}$ ANE & USA \\
\hline 2 & Sids 4 & MAYA" S"/ MON"S" / CMH74. A 592 / 3 / GIZA 157*2 & EGYPT \\
\hline 3 & Dovin - 2 & $\begin{array}{c}\text { CM } 84655-02 A P-300 \text { AP- } 300 L-3 \text { AP }-300 L-3 A P \\
- \text { OL - OAP }\end{array}$ & ICARDA \\
\hline 4 & Sids 1 & HD 21 / PAVON "S "// 1158. 57 / MAYA 74 "S" & EGYPT \\
\hline 5 & Gemmiza 10 & $\begin{array}{c}\text { MAYA } 74 \text { "S* } 10 \text { N/ } 1160-147 \text { / 3/ BB / G / LL / } 4 \text { / } \\
\text { CHAT " S" / } 5 \text { / CROW " S " }\end{array}$ & EGYPT \\
\hline 6 & Line \#3 & CHIL / SLM 75 & ICARDA \\
\hline 7 & Salha b1 & INIA / RL 4220 // 7 C / Y R " S " & EGYPT \\
\hline 8 & Sids 6 & MAYA "S" / MON "S" // CMH 74 A. 592 /3 /SAKHA 8* 2 & EGYPT \\
\hline 9 & Sakha 94 & Opta / RayoN // KAVZ & EGYPT \\
\hline 10 & Sahel 1 & NS 732 / PIMA / Vee "S" & EGYPT \\
\hline 11 & Line \#1 & $\begin{array}{c}\text { ALD / CEP } 75630 \text { // CCP } 75234 \text { / PT 7219/ } 3 \text { / BUC / } \\
\text { BJY / } 4 \text { / SARA // JUP / BJY / } 3 \text { / KAUZ / } 4 \text { / BABA X / } 5 \\
\text { / FRTL }\end{array}$ & ICARDA \\
\hline 12 & Line \# 2 & & ICARDA \\
\hline 13 & Giza 163 & T.AESTIVUM / BON // CNO / 7C & EGYPT \\
\hline 14 & Giza 170 & KAUZ // ALTAR 84 / AOS. & EGYPT \\
\hline 15 & Irina & CM $91575-284-O M-O Y-/ M-O Y$ & ICARDA \\
\hline 16 & Line \# & KANCHAN - OBGD & ICARDA \\
\hline 17 & PASTOR & CM 85295 - 0101 TOPY - 2M - OY - OM - OAP & ICARDA \\
\hline 18 & NABEK- 4 & ICW $94-0288-\mathrm{OL}-2$ AP $-2 \mathrm{AP}-\mathrm{OAPS}-\mathrm{OAP}$ & ICARDA \\
\hline \multicolumn{4}{|c|}{ Testers $(T)$} \\
\hline 1 & Gemmiza 9 & ALD "S" / HUAC "S" // CMH 74 A. 630 / SX & EGYPT \\
\hline 2 & Sakha 93 & SAKHA 92 / TR 810328 & EGYPT \\
\hline 3 & Giza 168 & MRL / BUC / SERI & EGYPT \\
\hline
\end{tabular}

In 2008 / 2009, the 21 parents along with the $54 \mathrm{~F} 1 / \mathrm{S}$ were grown in randomized complete block design with three replications. In 2009/2010 season the parents, $54 \mathrm{~F} 1$ populations and 54 F2 were grown in randomized complete block design with three replications. Each plot included two and six rows for F1 / F2 and parents, respectively. Rows were $4 \mathrm{~m}$ long and $20 \mathrm{~cm}$ apart and plants were spaced at $10 \mathrm{~cm}$ within row for each studied genotype. The recommended cultural practices were applied at the proper time. At maturity, ten guarded plants were randomly selected from each row and data were collected for number of spikes per plant, number of kernels per spike, kernel weight and grain yield per plant. Data for the characters depicting significant difference were further analyzed for line $\mathrm{x}$ tester according to Singh and Chaudhry (1979).

\section{RESULTS AND DISCUSSION}

The analysis of variance of combining ability for four characters showed that variances due to genotypes for F1 and F2 were highly significant (Table2), indicating that wheat genotypes differed in their genetic background. Parents vs. crosses mean 
squares, as an indication to average heterosis overall crosses, were found for most characters for $F 1$ and $F 2$ except for number of kernels per spike in $F 1$, indicating that the variance due to heterosis illustrating the wide range of heterosis values among the hybrids for all traits. Further partitioning of crosses mean squares i.e. line $\mathrm{x}$ tester analysis indicated that the difference due to both lines and testers were highly significant for all characters. The contribution of line $x$ tester interactions highly significant for all traits studied for F1 and F2 indicating that testers did not express similar ranking according to the performance of their crosses with the three testers. Also, the component of variation due to SCA was higher than GCA for all the characters in all the $F 1$ and $F 2$ indicating the predominance of non- additive gene action. Such results infers that the chosen material had high selection history. On the other hand, GCA/ SCA ratio was less than unity for all studied characters, indicating that SCA variance was more important than GCA variance. This indicant the predominantly non additive gene action for all traits in F1 and F2. The obtained results are in harmony with those previously reached by Hamada et. al. (2002) and Mohammad et al. (2009).

Table 2. Mean squares for all studied traits in F1 and F2 in wheat and their contribution to the total variation.

\begin{tabular}{|c|c|c|c|c|c|c|c|c|c|}
\hline \multirow{2}{*}{$\begin{array}{l}\text { Source of } \\
\text { variation }\end{array}$} & \multirow{2}{*}{$d f$} & \multicolumn{2}{|c|}{ No. of spikes/ plant } & \multicolumn{2}{|c|}{ No. of kernels/ spike } & \multicolumn{2}{|c|}{$\begin{array}{c}\text { 100- kernel weight } \\
\text { (g) }\end{array}$} & \multicolumn{2}{|c|}{$\begin{array}{c}\text { Grain yield/ plant } \\
\text { (g) }\end{array}$} \\
\hline & & F1 & F2 & $\mathrm{F} 1$ & F2 & $\mathrm{F} 1$ & F2 & F1 & F2 \\
\hline Replication & 2 & 4.85 & 4.99 & 35.03 & 16.03 & 0.17 & 0.01 & 10.75 & 2.00 \\
\hline Genotypes & 74 & $67.75^{* *}$ & $56.77^{* *}$ & $307.50 * *$ & $304.44 * *$ & $1.29 * *$ & $1.24 * *$ & $402.15^{* *}$ & $323.58^{* *}$ \\
\hline Parents $(\mathrm{P})$ & 20 & $62.20 * *$ & $62.20 * *$ & $384.49 * *$ & $384.50 * *$ & $1.61^{* *}$ & $1.62 * *$ & $279.29 * *$ & $279.30 * *$ \\
\hline Crosses (C) & 53 & $70.91^{* *}$ & $55.07 * *$ & $284.21 * *$ & $277.09 * *$ & $1.18^{* *}$ & $1.09 * *$ & $438.54 * *$ & $344.65^{* *}$ \\
\hline P.VS.C. & 1 & $11.05^{* *}$ & $37.89 * *$ & $1.28 \mathrm{NS}$ & $152.78^{* *}$ & $0.27 * *$ & $1.56 * *$ & $930.36 * *$ & $92.91^{* *}$ \\
\hline Lines $(\mathrm{L})$ & 17 & $151.64 * *$ & $114.43^{* *}$ & $53.05^{* *}$ & $570.30 * *$ & $2.56 * *$ & $2.31^{* *}$ & $1062.93 * *$ & $839.17 * *$ \\
\hline Testers $(T)$ & 2 & $54.15^{* *}$ & $42.67 * *$ & $14.28^{* *}$ & $47.09 * *$ & $0.33 * *$ & $0.33 * *$ & $49.87^{* *}$ & $33.79 * *$ \\
\hline LXT & 34 & $31.52^{* *}$ & $26.12^{* *}$ & $165.67 * *$ & $144.01^{* *}$ & $0.53 * *$ & $0.53 * *$ & $149.20 * *$ & $115.68^{* *}$ \\
\hline Error & 148 & 0.73 & 0.95 & 1.91 & 1.67 & 0.01 & 0.02 & 2.36 & 2.14 \\
\hline \multicolumn{2}{|c|}{ Variance due to GCA } & 0.42 & 0.31 & 1.27 & 1.42 & 0.007 & 0.006 & 3.09 & 2.45 \\
\hline \multicolumn{2}{|c|}{ Variance due to SCA } & 10.26 & 8.39 & 54.59 & 47.45 & 0.17 & 0.17 & 48.95 & 37.8 \\
\hline \multicolumn{2}{|c|}{$\begin{array}{c}\text { GCA / SCA Variance } \\
\text { ratio }\end{array}$} & 0.04 & 0.04 & 0.02 & 0.03 & 0.04 & 0.04 & 0.06 & 0.06 \\
\hline \multicolumn{10}{|c|}{ Proportional contribution to the total Variation } \\
\hline \multicolumn{2}{|c|}{ Contribution of lines } & 68.60 & 66.65 & 62.42 & 66.02 & 69.69 & 67.78 & 77.74 & 78.10 \\
\hline \multicolumn{2}{|c|}{ Contribution of tester } & 2.88 & 2.92 & 0.19 & 0.64 & 1.06 & 1.12 & 0.43 & 0.37 \\
\hline \multicolumn{2}{|c|}{ Contribution of $L X T$} & 28.52 & 30.42 & 37.39 & 33.34 & 29.25 & 31.10 & 21.83 & 21.53 \\
\hline
\end{tabular}

** Significant at $1 \%$ level of probability.

NS Not significant at $5 \%$ level of probability. 
The proportional contribution of lines (females), testers (males) and their interactions (Crosses) to total variance for different traits in F1 and F2 (Table2 )revealed that maximum contribution to total variance of grain yield per plant, number of spikes per plant, number of kernels per spike and kernel weight were made by female lines. Similar results were obtained by Hamada et al. 2002.

Table 3. Mean performance of F1 hybrids and F2 populations and their parents for all studied triats in a $18 \times 3$ line $X$ tester analysis in wheat

\begin{tabular}{|c|c|c|c|c|}
\hline Genotype & $\begin{array}{l}\text { No. of Spike/ } \\
\text { plant }\end{array}$ & $\begin{array}{l}\text { No. of kernels/ } \\
\text { spike }\end{array}$ & 100- kernels weight $(\mathrm{g})$ & $\begin{array}{c}\text { Grain yield/ plant } \\
\text { (g) }\end{array}$ \\
\hline F1 hybrid & & & & \\
\hline $\mathrm{L} 1 \mathrm{X}$ T1 & 20.61 & 70.93 & 4.36 & 39.67 \\
\hline T2 & 20.67 & 56.77 & 4.77 & 34.04 \\
\hline T3 & 19.09 & 78.97 & 4.50 & 51.63 \\
\hline L2X T1 & 12.10 & 75.03 & 4.90 & 24.57 \\
\hline $\mathrm{T} 2$ & 11.69 & 77.73 & 4.82 & 23.83 \\
\hline T3 & 11.31 & 75.17 & 4.73 & 30.34 \\
\hline $\mathrm{L} 3 \times \mathrm{T} 1$ & 29.67 & 69.83 & 4.59 & 65.07 \\
\hline T2 & 23.70 & 57.10 & 3.87 & 50.04 \\
\hline T3 & 24.75 & 58.50 & 3.93 & 68.80 \\
\hline L4 X T1 & 24.39 & 79.74 & 4.86 & 68.75 \\
\hline $\mathrm{T} 2$ & 21.69 & 68.84 & 3.39 & 58.07 \\
\hline T3 & 23.28 & 54.85 & 4.52 & 43.85 \\
\hline L5X T1 & 17.10 & 55.00 & 3.11 & 29.56 \\
\hline $\mathrm{T} 2$ & 16.88 & 71.70 & 4.68 & 34.68 \\
\hline T3 & 15.00 & 61.83 & 4.46 & 40.53 \\
\hline L6 X T1 & 21.45 & 51.10 & 5.59 & 33.49 \\
\hline $\mathrm{T} 2$ & 15.60 & 53.13 & 5.58 & 28.46 \\
\hline T3 & 21.07 & 50.45 & 5.52 & 30.60 \\
\hline L7 X T1 & 14.40 & 46.90 & 5.74 & 33.96 \\
\hline $\mathrm{T} 2$ & 11.63 & 66.13 & 6.07 & 24.84 \\
\hline T3 & 9.32 & 48.95 & 5.65 & 23.20 \\
\hline $\mathrm{L} 8 \times \mathrm{T} 1$ & 23.86 & 51.20 & 5.07 & 29.23 \\
\hline $\mathrm{T} 2$ & 14.93 & 48.17 & 5.67 & 27.82 \\
\hline T3 & 19.74 & 57.27 & 5.33 & 36.93 \\
\hline L9 X T1 & 20.84 & 60.33 & 5.32 & 41.54 \\
\hline $\mathrm{T} 2$ & 19.83 & 57.37 & 4.32 & 42.70 \\
\hline T3 & 25.03 & 64.77 & 4.62 & 46.99 \\
\hline $\mathrm{L} 10 \times \mathrm{T} 1$ & 12.74 & 69.97 & 5.36 & 30.34 \\
\hline $\mathrm{T} 2$ & 12.45 & 51.10 & 4.21 & 25.01 \\
\hline T3 & 13.92 & 55.13 & 4.22 & 27.57 \\
\hline L11 X T1 & 23.50 & 62.70 & 4.57 & 41.99 \\
\hline $\mathrm{T} 2$ & 13.13 & 72.23 & 4.76 & 29.73 \\
\hline T3 & 13.20 & 82.40 & 4.18 & 24.47 \\
\hline L12 X T1 & 21.87 & 59.87 & 5.43 & 38.93 \\
\hline $\mathrm{T} 2$ & 12.33 & 73.63 & 4.79 & 23.37 \\
\hline T3 & 12.27 & 60.83 & 4.34 & 26.53 \\
\hline L13 X T1 & 13.93 & 74.97 & 4.62 & 38.28 \\
\hline $\mathrm{T} 2$ & 18.67 & 70.03 & 4.79 & 43.32 \\
\hline T3 & 21.45 & 74.03 & 4.21 & 50.41 \\
\hline L14 X T1 & 18.08 & 77.03 & 4.47 & 48.84 \\
\hline $\mathrm{T} 2$ & 25.23 & 83.80 & 3.94 & 64.52 \\
\hline T3 & 18.08 & 68.47 & 4.30 & 52.13 \\
\hline L15 X T1 & 15.99 & 75.07 & 4.79 & 44.69 \\
\hline T2 & 18.38 & 79.83 & 5.23 & 61.63 \\
\hline T3 & 15.58 & 71.03 & 4.53 & 40.73 \\
\hline L16 X T1 & 19.03 & 63.38 & 4.49 & 45.63 \\
\hline $\mathrm{T} 2$ & 26.03 & 55.38 & 4.75 & 53.03 \\
\hline T3 & 14.00 & 68.07 & 5.08 & 44.53 \\
\hline
\end{tabular}


Table 3. Cont.

\begin{tabular}{|c|c|c|c|c|}
\cline { 1 - 3 } Genotype & \multirow{2}{*}{$\begin{array}{c}\text { No. of Spike/ } \\
\text { plant }\end{array}$} & $\begin{array}{c}\text { No. of kernels/ } \\
\text { spike }\end{array}$ & $\begin{array}{c}\text { 100- kernels } \\
\text { weight }(\mathrm{g})\end{array}$ & $\begin{array}{c}\text { Grain yield/ } \\
\text { plant }(\mathrm{g})\end{array}$ \\
\cline { 1 - 4 } F1 hybrid & 13.43 & 65.03 & 5.61 & 39.21 \\
\hline L17 X T1 & 12.55 & 64.83 & 5.23 & 36.48 \\
\hline T2 & 12.84 & 66.43 & 5.63 & 38.18 \\
\hline T3 & 13.43 & 66.53 & 5.33 & 41.81 \\
\hline T2 & 13.14 & 66.73 & 5.60 & 39.33 \\
\hline T3 & 12.55 & 61.43 & 5.71 & 37.39 \\
\hline MS (Genotypes) & $67.75^{* *}$ & $307.50^{* *}$ & $1.29^{* *}$ & $402.15^{* *}$ \\
\hline SE & 0.49 & 0.80 & 0.06 & 0.89 \\
\hline L.S.D at $1 \%$ & 1.82 & 2.95 & 0.22 & 3.29 \\
\hline
\end{tabular}

Table 3. Cont.

\begin{tabular}{|c|c|c|c|c|}
\hline $\begin{array}{l}\text { Genotype } \\
\text { F2 Crosses }\end{array}$ & $\begin{array}{l}\text { No. of spikes/ } \\
\text { plant }\end{array}$ & $\begin{array}{l}\text { No. of kernels/ } \\
\text { spike }\end{array}$ & $\begin{array}{l}\text { 100- kernels } \\
\text { weight }(\mathrm{g})\end{array}$ & $\begin{array}{l}\text { Grain yield/ plant } \\
(\mathrm{g})\end{array}$ \\
\hline L1 X T1 & 19.09 & 68.67 & 4.35 & 36.81 \\
\hline $\mathrm{T} 2$ & 19.23 & 55.87 & 4.60 & 31.58 \\
\hline T3 & 17.53 & 74.83 & 4.46 & 47.07 \\
\hline L2X T1 & 11.27 & 76.83 & 4.90 & 22.85 \\
\hline $\mathrm{T} 2$ & 10.88 & 77.83 & 4.86 & 22.13 \\
\hline T3 & 10.40 & 75.83 & 4.81 & 27.97 \\
\hline L3 X T1 & 27.03 & 64.67 & 4.50 & 58.92 \\
\hline $\mathrm{T} 2$ & 21.27 & 55.90 & 3.81 & 45.00 \\
\hline T3 & 22.29 & 54.87 & 3.92 & 61.90 \\
\hline L4 X T1 & 21.92 & 74.90 & 4.80 & 61.87 \\
\hline T2 & 19.51 & 66.97 & 3.36 & 52.30 \\
\hline T3 & 22.05 & 52.97 & 4.43 & 41.54 \\
\hline L5X T1 & 16.37 & 51.97 & 3.11 & 28.40 \\
\hline $\mathrm{T} 2$ & 16.20 & 69.03 & 4.51 & 33.23 \\
\hline T3 & 14.37 & 59.97 & 4.40 & 38.87 \\
\hline L6 X T1 & 19.66 & 50.00 & 5.50 & 30.64 \\
\hline $\mathrm{T} 2$ & 14.27 & 51.00 & 5.53 & 29.08 \\
\hline T3 & 19.30 & 49.47 & 5.39 & 27.96 \\
\hline L7 X T1 & 13.76 & 45.07 & 5.49 & 32.40 \\
\hline $\mathrm{T} 2$ & 11.10 & 63.23 & 5.88 & 23.63 \\
\hline T3 & 8.90 & 46.87 & 5.45 & 22.10 \\
\hline L8 X T1 & 22.01 & 49.45 & 4.95 & 26.94 \\
\hline $\mathrm{T} 2$ & 13.75 & 45.07 & 5.60 & 25.63 \\
\hline T3 & 18.20 & 55.37 & 5.09 & 34.03 \\
\hline L9 $\times$ T1 & 10.78 & 60.07 & 5.29 & 37.37 \\
\hline T2 & 17.81 & 56.07 & 4.24 & 38.31 \\
\hline T3 & 22.53 & 63.87 & 4.55 & 42.25 \\
\hline L10 X T1 & 11.96 & 67.97 & 5.30 & 28.33 \\
\hline T2 & 11.74 & 49.97 & 4.15 & 23.62 \\
\hline T3 & 13.10 & 53.97 & 4.19 & 26.03 \\
\hline L11 X T1 & 20.66 & 61.10 & 4.50 & 36.94 \\
\hline $\mathrm{T} 2$ & 11.53 & 71.83 & 4.60 & 26.03 \\
\hline T3 & 11.48 & 74.83 & 4.10 & 21.18 \\
\hline L12 X T1 & 19.70 & 58.17 & 5.30 & 35.02 \\
\hline $\mathrm{T} 2$ & 11.06 & 71.83 & 4.56 & 20.98 \\
\hline T3 & 10.98 & 59.23 & 4.08 & 23.86 \\
\hline L13 X T1 & 13.13 & 73.17 & 4.40 & 36.14 \\
\hline T2 & 17.86 & 68.83 & 4.68 & 40.99 \\
\hline T3 & 20.07 & 70.17 & 4.10 & 47.28 \\
\hline L14 X T1 & 16.12 & 70.17 & 4.20 & 43.43 \\
\hline T2 & 22.33 & 84.30 & 3.85 & 57.32 \\
\hline T3 & 16.01 & 65.67 & 4.19 & 46.30 \\
\hline L15 X T1 & 15.00 & 73.07 & 4.69 & 41.89 \\
\hline $\mathrm{T} 2$ & 17.24 & 80.03 & 5.09 & 57.76 \\
\hline T3 & 15.55 & 68.33 & 4.29 & 40.70 \\
\hline L16 X T1 & 17.10 & 60.53 & 4.39 & 41.03 \\
\hline T2 & 23.37 & 53.43 & 4.58 & 47.69 \\
\hline T3 & 12.64 & 63.97 & 4.99 & 40.05 \\
\hline
\end{tabular}


Table 3. Cont.

\begin{tabular}{|c|c|c|c|c|}
\hline Genotype & $\begin{array}{c}\text { No. of spikes/ } \\
\text { plant }\end{array}$ & $\begin{array}{c}\text { No. of kernels/ } \\
\text { spike }\end{array}$ & $\begin{array}{c}\text { 100- kernels } \\
\text { weight (g) }\end{array}$ & $\begin{array}{c}\text { Grain yield/ plant } \\
\text { (g) }\end{array}$ \\
\hline L17 X T1 & 12.70 & 64.77 & 5.38 & 37.05 \\
\hline T2 & 11.89 & 63.37 & 5.15 & 34.51 \\
\hline T3 & 12.15 & 65.17 & 5.55 & 36.15 \\
\hline L18 X T1 & 12.70 & 64.43 & 5.20 & 39.58 \\
\hline T2 & 12.44 & 64.27 & 5.40 & 37.21 \\
\hline T3 & 11.89 & 60.07 & 5.50 & 35.40 \\
\hline MS (Genotypes) & $56.77 * *$ & $304.44 * *$ & $1.24 * *$ & $323.58 * *$ \\
\hline SE & 0.56 & 0.75 & 0.08 & 0.84 \\
\hline L.S.D at 1\% & 2.07 & 2.77 & 0.30 & 3.10 \\
\hline
\end{tabular}

Table 3. Cont.

\begin{tabular}{|c|c|c|c|c|}
\hline Genotype & $\begin{array}{c}\text { No. of spikes/ } \\
\text { plant }\end{array}$ & No. of kernels/ spike & $\begin{array}{l}\text { 100- kernel } \\
\text { weight }(\mathrm{g})\end{array}$ & $\begin{array}{c}\text { Grain yield/ plant } \\
(\mathrm{g})\end{array}$ \\
\hline Line 1 & 17.93 & 72.63 & 5.44 & 38.47 \\
\hline 2 & 7.57 & 98.27 & 5.44 & 20.06 \\
\hline 3 & 18.72 & 68.83 & 5.12 & 49.17 \\
\hline 4 & 19.39 & 88.67 & 4.78 & 57.20 \\
\hline 5 & 16.65 & 75.67 & 4.98 & 27.25 \\
\hline 6 & 17.09 & 59.03 & 5.41 & 29.94 \\
\hline 7 & 26.47 & 54.33 & 5.29 & 28.06 \\
\hline 8 & 19.93 & 56.67 & 6.78 & 31.40 \\
\hline 9 & 17.23 & 67.27 & 5.18 & 39.77 \\
\hline 10 & 8.93 & 59.67 & 4.41 & 15.23 \\
\hline 11 & 14.43 & 56.67 & 4.88 & 28.55 \\
\hline 12 & 15.94 & 59.30 & 5.83 & 28.34 \\
\hline 13 & 23.73 & 62.03 & 4.88 & 47.56 \\
\hline 14 & 15.87 & 68.43 & 3.52 & 36.40 \\
\hline 15 & 16.38 & 57.27 & 4.07 & 32.48 \\
\hline 16 & 23.00 & 52.67 & 3.99 & 33.73 \\
\hline 17 & 13.02 & 62.07 & 5.68 & 38.26 \\
\hline 18 & 12.25 & 62.07 & 5.63 & 39.39 \\
\hline Tester 1 & 21.53 & 62.77 & 4.42 & 39.61 \\
\hline 2 & 15.23 & 59.87 & 4.30 & 37.55 \\
\hline 3 & 16.93 & 56.31 & 4.15 & 42.96 \\
\hline
\end{tabular}


Results in Table 3 further exhibited that genotypic and parental mean difference for all traits were significant at $1 \%$ level of significance. For number of spikes per plant , P7 genotype recorded the highest value (26.47).Moreover, crosses of L3 X T1 , L3 X T3, L9 X T3 , L14 X T2 and L16 X T2 gave the highest number of spike per plant $(29.67,27.03),(24.75,22.29),(25.03,22.53),(25.23,22.33)$ and $(26.03$, 23.37) for F1 and F2, respectively. Concerning number of kernels per spike, P2 recorded the highest number (98.27), but it was less in case of the hybrid L14 X T2 $(83.80,84.30)$ for $F 1$ and $F 2$, respectively. P8 gave the highest performance value of 100- kernels weight (6.78g) for F1 and F2, but it was less in case of the hybrid, L7 X $\mathrm{T} 2(6.07 \mathrm{~g}, 5.889)$ For F1and F2, respectively. Concerning grain yield per plant, data in Table3 show that the estimates of grain yield per plant was maximized in case of $L 3 x$ T3 (68.80, 61.90), L4 X T1 (68.75,61.879), L4 X T2 (58.07, 52.30), L14 X T2 (64.52, $57.32 \mathrm{~g})$ and L15 X T2 $(61.63,57.76 \mathrm{~g})$ for F1 and F2, respectively. So, the high desired values of the yield could be obtained from crosses between medium parents with low parents or from high parents for the aforementioned hybrids. It indicated that genotypes, parents, crosses and parents $\mathrm{X}$ crosses, all lines and testers markedly differ in combining ability effects for grain yield and all traits studied. Similar results were obtained by Muhammad et. al. (2009).

\section{Combining Ability Effects}

Estimates of both GCA and SCA for all traits in F1 and F2 are presented in Table 4 and Table 5, respectively. Both GCA and SCA effect were found to be highly significant for all characteristics studied. This shows the importance of additive and non- additive genetic variance in determining the performance of these traits. Therefore, it could be concluded that the selection procedures based on the accumulation of additive effect would be successful in improving these traits. However, selection advance procedures which are known to be effective in shifting gene frequency when additive and non- additive variances are involved should be maximized. Both significant GCA and SCA variance were found also for all traits studied by Hamada et. al. (2002) and Muhammad et. al. (2009). 
Table 4. General combining ability (GCA) effects of 18 lines and 3 testers for F1 and F2 in wheat.

\begin{tabular}{|c|c|c|c|c|c|c|c|c|}
\hline \multirow{2}{*}{$\begin{array}{l}\text { Character } \\
\text { Genotypic }\end{array}$} & \multicolumn{2}{|c|}{ No. of spike/ plant } & \multicolumn{2}{|c|}{ No. of kernels/ spike } & \multicolumn{2}{|c|}{$\begin{array}{l}\text { 100- kernels weight } \\
\text { (g) }\end{array}$} & \multicolumn{2}{|c|}{$\begin{array}{c}\text { Grain yield/ plant } \\
\text { (g) }\end{array}$} \\
\hline & $\mathrm{F} 1$ & $\mathrm{~F} 2$ & $\mathrm{~F} 1$ & $\mathrm{~F} 2$ & $\mathrm{~F} 1$ & $\mathrm{~F} 2$ & $\mathrm{~F} 1$ & $\mathrm{~F} 2$ \\
\hline Line 1 & 2.63 & 2.47 & $3.93 * *$ & $3.51 * *$ & $-0.27 * *$ & $-0.24 * *$ & $1.94 * *$ & $1.75^{* *}$ \\
\hline 2 & $-5.85 * *$ & $-5.30 * *$ & $11.02 * *$ & $13.88 * *$ & $0.003 * *$ & $0.15^{* *}$ & $-13.58 * *$ & $-12.42 * *$ \\
\hline 3 & $8.48 * *$ & $7.39 * *$ & $-3.14 * *$ & $-4.47 * *$ & $-0.68 * *$ & $-0.63 * *$ & $21.47 * *$ & $18.54 * *$ \\
\hline 4 & $5.56 * *$ & $5.01 * *$ & $2.85 * *$ & $2.00 * *$ & $-0.55^{* *}$ & $-0.51 * *$ & $17.05^{* *}$ & $15.17 * *$ \\
\hline 5 & $-1.22 * *$ & $-0.50 * *$ & $-2.11 * *$ & $-2.63 * *$ & $-0.76 * *$ & $-0.70 * *$ & $-4.90 * *$ & $-3.23 * *$ \\
\hline 6 & $1.82 * *$ & $1.60 * *$ & $-13.39 * *$ & $-12.79 * *$ & $0.74 * *$ & $0.77 * *$ & $-8.98 * *$ & $-7.51 * *$ \\
\hline 7 & $-5.76 * *$ & $-4.69 * *$ & $-10.96 * *$ & $-11.22 * *$ & $0.99 * *$ & $0.90 * *$ & $-12.50 * *$ & $-10.69 * *$ \\
\hline 8 & $1.95 * *$ & $1.84 * *$ & $-12.74 * *$ & $-12.98 * *$ & $0.54 * *$ & 0.50 & $-8.50 * *$ & $-7.87 * *$ \\
\hline 9 & $4.35 * *$ & 3.56 & $-4.13 * *$ & $-2.95^{* *}$ & $-0.06 * *$ & $-0.01 * *$ & $3.91 * *$ & $2.58 * *$ \\
\hline 10 & $-4.51 * *$ & $-3.88 * *$ & $-6.22 * *$ & $-5.65 * *$ & $-0.22 * *$ & $-0.16^{* *}$ & $-12.29 * *$ & $-10.74 * *$ \\
\hline 11 & $-0.94 * *$ & $-1.59 * *$ & 7.48 & 6.31 & $-0.31 * *$ & $-0.31 * *$ & $-7.76 * *$ & $-8.55^{* *}$ \\
\hline 12 & $-2.06 * *$ & $-2.23 * *$ & $-0.98 * *$ & $0.13 * *$ & $0.03 * *$ & $-0.05^{* *}$ & $-10.22 * *$ & $-10.12 * *$ \\
\hline 13 & $0.53 * *$ & $0.88 * *$ & $8.05^{* *}$ & $7.77 * *$ & $-0.27 * *$ & $-0.31 * *$ & $4.16^{* *}$ & 4.74 \\
\hline 14 & 2.91 & 2.01 & $11.47 * *$ & $10.43 * *$ & $-0.58 * *$ & $-0.63 * *$ & $15.33 * *$ & $12.29 * *$ \\
\hline 15 & $-0.89 * *$ & $-0.22 * *$ & $10.35^{* *}$ & $10.86 * *$ & $0.03 * *$ & $-0.02 * *$ & $9.18 * *$ & $10.05^{* *}$ \\
\hline 16 & $2.13 * *$ & $1.55^{* *}$ & $-2.68 * *$ & $-3.64 * *$ & $-0.04 * *$ & $-0.05^{* *}$ & $7.90 * *$ & $6.19 * *$ \\
\hline 17 & $-4.61 * *$ & $-3.90 * *$ & $0.47 * *$ & $1.48 * *$ & $0.67 * *$ & $0.65 * *$ & $-1.87 * *$ & $-0.83 * *$ \\
\hline 18 & $-4.51 * *$ & $-3.80 * *$ & $-0.05 * *$ & $-0.03 * *$ & $0.73 * *$ & $0.66 * *$ & $-0.32 * *$ & $0.66^{* *}$ \\
\hline Tester 1 & 1.13 & 1.02 & 0.29 & 0.11 & 0.08 & 0.08 & 1.01 & 0.80 \\
\hline 2 & -0.38 & -0.40 & 0.29 & 0.87 & -0.01 & -0.03 & -0.89 & -0.78 \\
\hline 3 & $-0.74 * *$ & $-0.62 * *$ & -0.59 & -0.98 & -0.06 & -0.07 & -0.12 & -0.02 \\
\hline $\begin{array}{c}\text { SE of GCA } \\
\text { lines }\end{array}$ & 0.29 & 0.32 & 0.46 & 0.43 & 0.04 & 0.04 & 0.51 & 0.49 \\
\hline $\begin{array}{c}\text { SE of GCA } \\
\text { testes }\end{array}$ & 0.12 & 0.13 & 0.19 & 0.18 & 0.02 & 0.02 & 0.21 & 0.20 \\
\hline
\end{tabular}

** Significant at $1 \%$ level of probability.

\section{General Combining Ability Effects (GCA).}

Results in Table 4 show the estimates of general combining ability (GCA) effects were highly significant for most parents in F1 and F2 for all studied traits. High positive values of general combining ability effects would be of interest in all traits. The results revealed that lines number $3,4,9$, and 16 had significantly greater positive values of 
GCA for number of spike per plant than the other parents, that these cultivars are good donors for improving this trait. Meantime, wheat lines 2, 11, 13, 14 and 15 showed desirable general combining ability effects for number of kernels per spike, while lines number $6,7,8,17$ and 18 for 100- kernels weight. Obviously, wheat lines number 3, 4, 14,15 and 16 were good donors for grain yield per plant. The tester cultivar number 1 proved to be good general combiner for all traits. For GCA effects of females and males, four lines number 3,4,14 and 15 proved as better general combiners for grain yield per plant and most of other traits, which are important components in grain yield improvement. These parents also contributed a large number of favorable genes for high grain yield per plant and other related traits. Hence, four female parents could be used in future programs to improve grain yield.

Tester number 1 appeared to be better general combiner for grain yield per plant and some other traits. These parents presumed to have a relatively large number of favorable alleles for these traits.

Table 5. Specific combining ability of crosses developed from 18 lines and 3 testers for $\mathrm{F} 1$ and $\mathrm{F} 2$ in wheat.

\begin{tabular}{|c|c|c|c|c|c|c|c|c|}
\hline \multirow{2}{*}{$\begin{array}{l}\text { Character } \\
\text { Genotype }\end{array}$} & \multicolumn{2}{|c|}{ No. of spike/ plant } & \multicolumn{2}{|c|}{ No. of kernels/ spike } & \multicolumn{2}{|c|}{100 - kernels weight(g) } & \multicolumn{2}{|c|}{ Grain yield/ plant } \\
\hline & $\mathrm{F} 1$ & $\mathrm{~F} 2$ & $\mathrm{~F} 1$ & $\mathrm{~F} 2$ & $\mathrm{~F} 1$ & $\mathrm{~F} 2$ & $\mathrm{~F} 1$ & $\mathrm{~F} 2$ \\
\hline $\mathrm{L} 1 \times \mathrm{T} 1$ & $-0.71 * *$ & $-0.55 * *$ & $1.74 * *$ & $2.10 * *$ & $-0.27 * *$ & $-0.20 * *$ & $-3.12 * *$ & $-2.48 * *$ \\
\hline $\mathrm{T} 2$ & 1.06 & 1.01 & $-12.41^{* *}$ & $-11.46 * *$ & 0.24 & 0.14 & $-6.84 * *$ & $-6.12 * *$ \\
\hline T3 & $-0.34 * *$ & $-0.46 * *$ & $10.67 * *$ & $9.36 * *$ & $0.02 * *$ & $0.06 * *$ & $9.97 * *$ & $8.60 * *$ \\
\hline $\mathrm{L} 2 \times \mathrm{T} 1$ & $-0.73 * *$ & $-0.60 * *$ & $-1.24 * *$ & $-0.11 * *$ & $-0.003 * *$ & $-0.04 * *$ & $-2.69 * *$ & $-2.26 * *$ \\
\hline $\mathrm{T} 2$ & $0.37 * *$ & 0.43 & $1.46 * *$ & $0.13 * *$ & $0.02 * *$ & $0.02 * *$ & $-1.52 * *$ & $-1.40 * *$ \\
\hline T3 & $0.35^{* *}$ & $0.17 * *$ & $-0.21 * *$ & $-0.02 * *$ & $-0.01 * *$ & $0.02 * *$ & 4.21 & 3.66 \\
\hline $\mathrm{L} 3 \times \mathrm{T} 1$ & 2.49 & 2.48 & $7.72 * *$ & 6.08 & 0.37 & 0.34 & 2.74 & 2.84 \\
\hline $\mathrm{T} 2$ & $-1.94 * *$ & $-1.87 * *$ & $-5.00 * *$ & $-3.45^{* *}$ & $-0.24 * *$ & $-0.25^{* *}$ & $-10.36 * *$ & $-9.49 * *$ \\
\hline T3 & $-0.54 * *$ & $-0.62 * *$ & $-2.71 * *$ & $-2.63 * *$ & $-0.13 * *$ & $-0.09 * *$ & $7.61^{* *}$ & $6.65^{* *}$ \\
\hline $\mathrm{L} 4 \times \mathrm{T} 1$ & $0.13^{* *}$ & $-0.26 * *$ & $11.63 * *$ & $9.85 * *$ & $0.52 * *$ & $0.52 * *$ & $10.84 * *$ & $9.17 * *$ \\
\hline $\mathrm{T} 2$ & $-1.04 * *$ & $-1.25^{* *}$ & $0.73 * *$ & $1.15^{* *}$ & $-0.85 * *$ & $-0.83 * *$ & 2.07 & $1.18^{* *}$ \\
\hline T3 & 0.90 & 1.51 & $-12.37 * *$ & $-11.00 * *$ & 0.32 & 0.30 & $-12.91^{* *}$ & $-10.35^{* *}$ \\
\hline L5X T1 & $-0.36 * *$ & $-0.30 * *$ & $-8.14 * *$ & $-8.45^{* *}$ & $-1.02 * *$ & $-0.98^{* *}$ & $-6.37 * *$ & $-5.90 * *$ \\
\hline $\mathrm{T} 2$ & 0.94 & 0.95 & $8.56 * *$ & $7.84 * *$ & $0.54 * *$ & $0.52 * *$ & $0.64 * *$ & $0.51^{* *}$ \\
\hline T3 & $-0.57 * *$ & $-0.66 * *$ & $-0.41 * *$ & $0.63 * *$ & $0.47 * *$ & $0.47 * *$ & 5.72 & 5.39 \\
\hline $\mathrm{L} 6 \times \mathrm{T} 1$ & 0.93 & 0.90 & $-0.75^{* *}$ & $-0.26 * *$ & $-0.05^{* *}$ & $-0.06 * *$ & $1.62^{* *}$ & $0.61^{* *}$ \\
\hline $\mathrm{T} 2$ & $-3.38 * *$ & $-3.08 * *$ & $1.27 * *$ & $-0.03 * *$ & $0.03 * *$ & 0.07 & $-1.43 * *$ & $0.64 * *$ \\
\hline $\mathrm{T} 3$ & 2.44 & 2.18 & $-0.51 * *$ & $0.29 * *$ & $0.02 * *$ & $-0.01 * *$ & $-0.12 * *$ & $-1.25^{* *}$ \\
\hline L7 X T1 & 1.48 & 1.49 & $-7.39 * *$ & $-6.76 * *$ & $-0.15^{* *}$ & $-0.20 * *$ & 5.61 & 5.56 \\
\hline $\mathrm{T} 2$ & 0.23 & 0.25 & $11.84 * *$ & $10.64 * *$ & 0.24 & 0.29 & $-1.60 * *$ & $-1.63 * *$ \\
\hline $\mathrm{T} 3$ & $-1.71^{* *}$ & $-1.74 * *$ & $-4.45^{* *}$ & $-3.87 * *$ & $-0.08 * *$ & $-0.09 * *$ & $-4.01 * *$ & $-3.93 * *$ \\
\hline $\mathrm{L} 8 \times \mathrm{T} 1$ & 3.21 & 3.01 & $-1.30 * *$ & $-0.61 * *$ & $-0.37 * *$ & $-0.35^{* *}$ & $-3.10 * *$ & $-2.72 * *$ \\
\hline $\mathrm{T} 2$ & $-4.18^{* *}$ & $-3.84 * *$ & $-4.33 * *$ & $-5.77 * *$ & 0.33 & 0.40 & $-2.61 * *$ & $-2.46^{* *}$ \\
\hline T3 & 0.97 & 0.83 & 5.64 & $6.38 * *$ & $0.03 * *$ & $-0.05^{* *}$ & 5.71 & 5.18 \\
\hline L9 X T1 & $-2.19 * *$ & $-1.94 * *$ & $-0.79 * *$ & $-0.04 * *$ & $0.48^{* *}$ & 0.51 & $-3.21 * *$ & $-2.74 * *$ \\
\hline $\mathrm{T} 2$ & $-1.68 * *$ & $-1.50 * *$ & $-3.74 * *$ & $-4.81 * *$ & $-0.41 * *$ & $-0.44 * *$ & $-0.15^{* *}$ & $-0.21 * *$ \\
\hline $\mathrm{T} 3$ & $3.87 * *$ & $3.45 * *$ & $4.53 * *$ & 4.85 & $-0.06 * *$ & $-0.08^{* *}$ & 3.36 & 2.96 \\
\hline $\mathrm{L} 10 \times \mathrm{T} 1$ & $-1.43 * *$ & $-1.33 * *$ & $10.93 * *$ & $10.56 * *$ & $0.67 * *$ & $0.67 * *$ & $1.48^{* *}$ & 1.54 \\
\hline $\mathrm{T} 2$ & $-0.19 * *$ & $-0.13 * *$ & $-7.92 * *$ & $-8.21 * *$ & $-0.37 * *$ & $-0.38 * *$ & $-1.63 * *$ & $-1.59 * *$ \\
\hline T3 & 1.63 & 1.45 & $-3.00 * *$ & $-2.35^{* *}$ & $-0.30 * *$ & $-0.29 * *$ & $0.15^{* *}$ & $0.05^{* *}$ \\
\hline
\end{tabular}


Table 5. Cont.

\begin{tabular}{|c|c|c|c|c|c|c|c|c|}
\hline \multirow{2}{*}{$\begin{array}{l}\text { Character } \\
\text { Genotype }\end{array}$} & \multicolumn{2}{|c|}{ No. of spike/ plant } & \multicolumn{2}{|c|}{$\begin{array}{l}\text { No. of kernels/ } \\
\text { spike }\end{array}$} & \multicolumn{2}{|c|}{$\begin{array}{c}\text { 100- kernels weight } \\
\text { (g) }\end{array}$} & \multicolumn{2}{|c|}{$\begin{array}{c}\text { Grain yield/ plant } \\
\text { (g) }\end{array}$} \\
\hline & F1 & $\mathrm{F} 2$ & $\mathrm{~F} 1$ & F2 & $\mathrm{F} 1$ & F2 & $\mathrm{F} 1$ & F2 \\
\hline L11 X T1 & $5.75 * *$ & $5.09 * *$ & $\begin{array}{c}- \\
10.04 * *\end{array}$ & $-8.26 * *$ & $-0.02 * *$ & $0.02 * *$ & $8.91 * *$ & $7.95^{* *}$ \\
\hline $\mathrm{T} 2$ & -3.08 & $-2.63 * *$ & $-0.50 * *$ & $1.70 * *$ & 0.27 & 0.22 & $-1.43 * *$ & $-1.26 * *$ \\
\hline T3 & $-2.66 * *$ & $-2.45^{* *}$ & $10.54 * *$ & $6.56 * *$ & $-0.25 * *$ & $-0.23 * *$ & $7.47 * *$ & $-6.70 * *$ \\
\hline $\mathrm{L} 12 \times \mathrm{T} 1$ & $-5.24 * *$ & $4.77 * *$ & $-5.20 * *$ & $-5.02 * *$ & $0.49 * *$ & $0.56 * *$ & $8.30 * *$ & $7.60 * *$ \\
\hline $\mathrm{T} 2$ & $-2.76 * *$ & $-2.46 * *$ & $8.56 * *$ & $7.88^{* *}$ & $-0.04 * *$ & $-0.06 * *$ & $-5.35 * *$ & $-4.86 * *$ \\
\hline T3 & $-2.47 * *$ & $-2.31 * *$ & $-3.35 * *$ & $-2.86 * *$ & $-0.44 * *$ & $-0.50 * *$ & $-2.95 * *$ & $-2.74 * *$ \\
\hline $\mathrm{L} 13 \times \mathrm{T} 1$ & $-5.29 * *$ & $-4.91 * *$ & $1.65 * *$ & $2.34 * *$ & $-0.001 * *$ & $-0.08 * *$ & $-6.73 * *$ & $-6.13 * *$ \\
\hline $\mathrm{T} 2$ & 1.18 & 1.24 & $-3.27 * *$ & $-2.76 * *$ & 0.26 & 0.30 & $0.21 * *$ & $0.31^{* *}$ \\
\hline T3 & $4.16 * *$ & $3.67 * *$ & $1.61^{* *}$ & $0.43 * *$ & $-0.26 * *$ & $-0.23 * *$ & 6.52 & 5.83 \\
\hline $\mathrm{L} 14 \times \mathrm{T} 1$ & $-3.51 * *$ & $-3.06 * *$ & $0.30 * *$ & $-3.32 * *$ & 0.14 & $0.03 * *$ & $-7.33 * *$ & $-6.83 * *$ \\
\hline $\mathrm{T} 2$ & $5.15^{* *}$ & $4.58^{* *}$ & $7.07 * *$ & $10.05^{* *}$ & $-0.27 * *$ & $-0.22 * *$ & $10.25 * *$ & $9.08^{* *}$ \\
\hline T3 & $-1.63 * *$ & $-1.52 * *$ & $-7.37 * *$ & $-6.73 * *$ & 0.13 & 0.18 & $-2.91 * *$ & $-2.70 * *$ \\
\hline L15X T1 & $-1.79 * *$ & $-1.95 * *$ & $-0.54 * *$ & $-0.85 * *$ & $-0.14 * *$ & $-0.08 * *$ & $-5.34 * *$ & $-5.69 * *$ \\
\hline T2 & 2.11 & 1.71 & 4.22 & $5.35^{* *}$ & 0.39 & 0.41 & $13.50 * *$ & $11.76 * *$ \\
\hline T3 & $-0.32 * *$ & $0.24 * *$ & $-3.68 * *$ & $-4.50 * *$ & $-0.25^{* *}$ & $-0.33 * *$ & $-8.16^{* *}$ & $-6.07 * *$ \\
\hline L16 X T1 & $-1.79 * *$ & $-1.62 * *$ & $0.80 * *$ & $1.12 * *$ & $-0.37 * *$ & $-0.35 * *$ & $-3.11^{* *}$ & $-2.69 * *$ \\
\hline $\mathrm{T} 2$ & $6.73 * *$ & $6.06 * *$ & $-7.18^{* *}$ & $-6.75 * *$ & $-0.001 * *$ & $-0.06 * *$ & $-6.19 * *$ & 5.55 \\
\hline T3 & $-4.93 * *$ & $-4.44 * *$ & 6.38 & 5.64 & 0.37 & 0.41 & $-3.07 * *$ & $-2.86 * *$ \\
\hline L17 X T1 & $-0.64 * *$ & $-0.57 * *$ & $-0.69 * *$ & $0.23 * *$ & $0.02 * *$ & $-0.06 * *$ & $0.23 * *$ & $0.35^{* *}$ \\
\hline $\mathrm{T} 2$ & $-0.01 * *$ & $0.04 * *$ & $-0.89 * *$ & $-1.94 * *$ & $-0.24 * *$ & $-0.20 * *$ & $-0.58 * *$ & $-0.61 * *$ \\
\hline $\mathrm{T} 3$ & 0.65 & 0.52 & $1.59 * *$ & $1.71 * *$ & 0.21 & 0.26 & $0.34 * *$ & $0.26 * *$ \\
\hline L18 X T1 & $-0.74 * *$ & $-0.67 * *$ & $1.33 * *$ & $1.40 * *$ & $-0.30 * *$ & $-0.25 * *$ & $1.28 * *$ & $1.39 * *$ \\
\hline $\mathrm{T} 2$ & $0.48 * *$ & 0.49 & $1.53 * *$ & $0.47 * *$ & 0.07 & $0.05^{* *}$ & $0.71 * *$ & $0.59 * *$ \\
\hline T3 & $0.25^{* *}$ & $0.17 * *$ & -2.87 & $-1.87 * *$ & 0.23 & 0.20 & $-2.00 * *$ & $-1.98 * *$ \\
\hline $\begin{array}{l}\text { SE(SCA } \\
\text { effect) }\end{array}$ & 0.49 & 0.56 & 0.80 & 0.75 & 0.07 & 0.07 & 0.89 & 0.85 \\
\hline $\begin{array}{l}\text { SE (gi-Gj) for } \\
\text { lines }\end{array}$ & 0.40 & 0.46 & 0.65 & 0.61 & 0.06 & 0.06 & 0.73 & 0.69 \\
\hline $\begin{array}{l}\text { SE (gi-Gj) for } \\
\text { tester }\end{array}$ & 0.16 & 0.19 & 0.27 & 0.25 & 0.02 & 0.02 & 0.30 & 0.28 \\
\hline $\begin{array}{c}\text { SE (S ij - S } \\
k j)\end{array}$ & 0.70 & 0.79 & 1.13 & 1.05 & 0.10 & 0.10 & 1.26 & 1.20 \\
\hline
\end{tabular}

** Significant at $1 \%$ level of significance.

\section{Specific Combining Ability (SCA) effects}

Data presented in Table 5 showed that most hybrids exhibited significant and positive specific combining hybrids exhibited ability effects for yield and yield components trait. Seven crosses (L1xT3), (L3 x T3), (L4 x T1), (L11 x T1), (L12 xT1), (L14 x T2) and (L15 x T2) were observed as good specific combiners for grain yield per plant and some other traits. It could be concluded that the parental lines L3, L4, L14 and L15 might be selected as parental materials for wheat breeding programmes since the lines number 3, 4, 14 and 15 had the highest general combining ability effect for grain 
yield per plant and some other traits. Other crosses had worthless to mention because these were found as poor combiner especially for grain yield per plant .Better specific combining crosses might involved four good general combining parents, but this is not a rule for all cross. It can be concluded that crosses (L3 x T3), (L4 x T1), (L14 x T2) and (L15 x T2) were excellent harmonious combinations for SCA effects for all traits including grain yield per plant. It would yield transgressive segregants for grain yield plant in late segregating generations. Mass selection would be effective in late segregating generations in these crosses due to predominant non - additive gene action being operated for all traits. Such results were in agreement with those reported by Hamada et. al. (2002) and Muhammad et al. (2009). From the above - mentioned results it could be suggested that one or more of these hybrids might be in corporated in wheat breeding program to improve grain yield and its components.

\section{REFERENCES}

1. Ahmed, J. A. A. Z., B. Y. Samadi, A. Talaie, M. R. Channadha and A. Saeidi. 2003. A study of combining ability and gene effect in bread wheat under drought stress conditions by diallel method. Iranian J.Agri. Sci. 34: 1-8.

2. Gupta, R. R., and Z. Ahmed 1995. Line X tester analysis for some metric traits in macroni wheat. Crop Improvement, 22 (2): 224-249.

3. Hamada, A. A., E.H.Seidy and H. I. Hendawy. 2002. Breeding measurements for heading date, yield and yield components in wheat using line $X$ tester analysis. Annals Agric. Sci., Ain Shams Univ, Cairo.47(2), 587-609.

4. Hassan, E. E. and A. M. Abd El- Moniem. 1991. Combining ability, heterosis and gene action of some quantitave characters in wheat. Zagazig J. Agric. Res. 18(5): $1369-1381$.

5. Joshi S. K., S. N. Sharma, D. L. Singhania and R. S. Sain.2003.Genetic analysis of yield and its component traits in spring wheat, Triticum aestivum L. Acta Agronomica Hungarica.51: 139- 147.

6. Masood, M. S. and W. E. Kronstad. 2000. Combining ability analysis over various generations in a diallel cross of bread wheat. Pak. J.Agric. Res. 16:1-4.

7. Muhammad, A., J. Anwar, M. Hussain, M. H. Qureshi and S. Khan. 2009. Line X tester analysis in bread wheat (Triticum aestivum L.). J.Agric. Res. 47 (1): 411420.

8. Rajara, M. P. and R. V. Maheshwari. 1996. Combining ability in wheat using line $x$ tester analysis. Madras, Agric. J. 33(2): 107-110. 
9. Salem, A. H. and E. E. Hassan. 1991. Estimates of some breeding Parameters for yield and its attributes in wheat using line $x$ tester analysis. Zagazig J. Agric. Res, 18 (5): 1357-1368.

10. Sing, R. K. and B. D. Chaudhry. 1979. Biometrical methods in quantitative genetics analysis. 191-199. $2^{\text {nd }}$ Ed. Kalyan: Publishers, New Delhi, India.

11. Singh, B. D., P. K. Majumdar and K. K. Prasad. 2000. Combining ability for yield and its components in late sown wheat. J. Appl. Biol.10:119-126.

12. Singh, R. P., I. Singh, S. C. Sharman and L. D. Tripathi. 1994. Impact of Genetic background of testers on some biometrical Parameters in wheat (Triticum aestivum L.). Indian J.Agric.Res.28 (2):93-98.

13. Suleyman, S. and N. Akguni. 2007. Combining ability and inheritance of some agronomic traits in bread wheat (Triticum aestivium L.). Ziraat Fakultesi Dergisi. 21(41): 104-108. 


\section{تحليل السلالة X الكثاف للمحصول \\ ومكوناته فى قمح الخبز}

نادية عدلى رياض عبد النور 1 ، هيام سبد أحمد فاتح² أحمد كمال مصطفى1

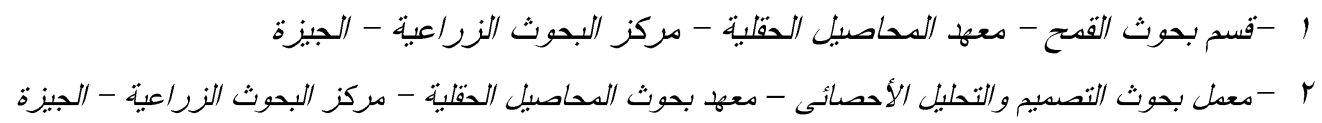

أجرى هذا البحث بمزرعة محطة البحوث الزر اعية بالجيزة خلال موسمى 2007 /2008 و2009/2008 حيث أجريت التهجينات اللازمة بأستخدام 18 نركيب وراثى كأمهات وثلاثة أباء

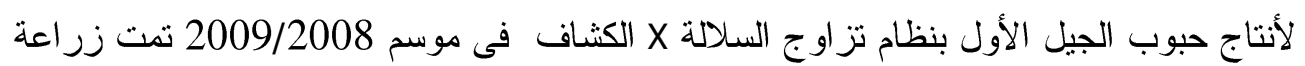
الحبوب الهجينة لأنتاج نباتات الجيل الأول وفى موسم 2009 /2010 نم أجرى التقيم النهائى للتجربة فى محطة البحوث الزر اعية بكفر الحمام محافظة الثرقية حيث تمت زر اعة حبوب الجيل ملاجل

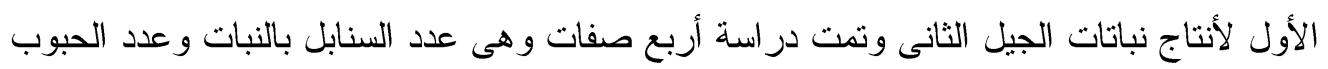
بالسنبلة ووزن الحبة ومحصول الحبوب بالنبات وذلك بغرض در اسة قوة الهجين و القدرة على التألف و الفعل الجينى المتحكم فى المحصول وصنفاتة .

أوضحت النتائج الدور المهم لكل من تأثير الفعل الجينى المضيف و الفعل الجينى غير وهن

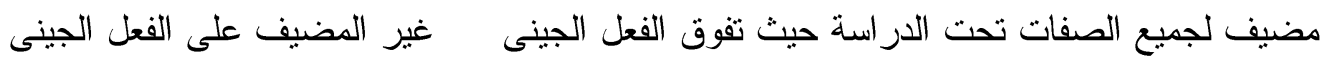

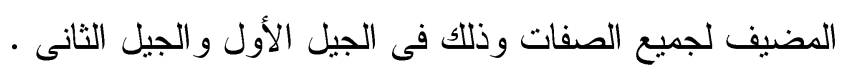
كانت السلالات 3, 14, 15 أفضل السلالات للأستخدام فى بر امج التربية لقدر اتها العالية

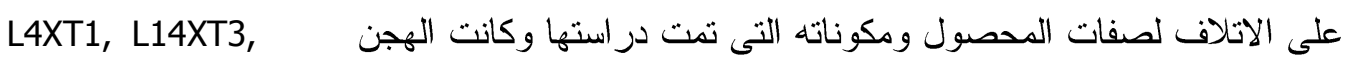
L3XT3， L15XT2 الاتلاف للمحصول ومكوناته. 